\title{
Identification of the TTC26 Splice Variant in a Novel Complex Ciliopathy Syndrome with Biliary, Renal, Neurological, and Skeletal Manifestations
}

\author{
Majid Alfadhela,b,c Muhammad Umair ${ }^{a, b} \quad$ Bader Almuzzainia,b Abdulaziz Asiria, e \\ Abeer Al Tuwaijri ${ }^{a, b}$ Khaloud Alhamoudi ${ }^{a, b}$ Yusra Alyafee $^{a, b}$ \\ Mohammed Al-Owain ${ }^{d}$
}

\begin{abstract}
aMedical Genomics Research Department, King Abdullah International Medical Research Center, Ministry of National

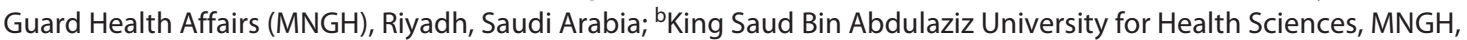
Riyadh, Saudi Arabia; 'Division of Genetics, Department of Pediatrics, King Abdullah Specialized Children's Hospital, King Abdulaziz Medical City, Riyadh, Saudi Arabia; ${ }^{d}$ Department of Genetics, King Faisal Specialist Hospital and Research Center, Riyadh, Saudi Arabia; ${ }^{\mathrm{e} F a c u l t y}$ of Applied Medical Sciences, University of Bisha, Bisha, Saudi Arabia
\end{abstract}

\section{Keywords}

TTC26 · Ciliopathy $\cdot$ Hydrocephalus $\cdot$ Cholestasis $\cdot$ Splice site variant

\begin{abstract}
Ciliopathies constitute heterogeneous disorders that result from mutations in ciliary proteins. These proteins play an important role in the development of organs, physiology, and signaling pathways, and sequence variations in the genes encoding these proteins are associated with multisystem disorders. In this study, we describe a severe ciliopathy disorder that segregates in an autosomal recessive manner in a nonconsanguineous Saudi family. The proband exhibited features such as cholestasis, cystic dilatation of intrahepatic biliary ducts, diabetes insipidus, dysmorphic facial features, optic atrophy, pituitary hypoplasia, hydrocephalus, aqueductal stenosis, hyperextensible knee joints, bilateral knee dislocation, polydactyly, and syndactyly. Whole-genome sequencing and Sanger sequencing revealed a homozygous splice site variant (c.4-1G>C; NM_024926.3) in the tetratricopeptide repeat domain 26 (TTC26) gene located in chromo-
\end{abstract}

some 7q34, which cosegregated perfectly with the disease phenotype. qRT-PCR revealed a substantial decrease in the expression of the $\Pi$ TC26 gene as compared to the normal control, suggesting the pathogenicity of the identified variant. This report further strengthens the evidence that homozygous variants in the TTC26 gene cause severe ciliopathies with diverse phenotypes. We named this newly characterized condition as BRENS syndrome, which stands for biliary, renal, neurological, and skeletal features.

(c) 2021 S. Karger AG, Basel

\section{Introduction}

Ciliopathies constitute a heterogeneous group of disorders associated with dysfunctions of the cilia. Many ciliary proteins have been reported to play an important role in human development, physiology, and signaling pathways, and are also associated with severe multisystem disorders [Reiter and Leroux, 2017]. Defects in the primary cilia have severe clinical consequences with diverse phenotypic variations that are collectively termed as ciliopa- 
thies [Shaheen et al., 2016]. These ciliopathies cause several human diseases, such as polycystic kidney, primary ciliary dyskinesia, Bardet-Biedl syndrome, Joubert syndrome, and Meckel-Gruber syndrome [Hildebrandt et al., 2011; Shaheen et al., 2016].

The maintenance and assembly of cilia are regulated by the intraflagellar transport (IFT) along the ciliary axoneme. IFT is mediated by the bi-directional movement of several protein complexes called IFT proteins [Scholey, 2008; Ishikawa and Marshall, 2011; Ishikawa et al., 2014].

Tetratricopeptide repeat domain 26 (TTC26; OMIM 617453) is a homolog of Trypanosoma brucei PIFTC3 and Caenorhabditis elegans DYF-13. In C. elegans dye-filling defective (dyf)-13 mutants, defects in ciliary assembly have been observed, suggesting that TTC26/DYF13 is a recognized IFT protein [Follit et al., 2009; Franklin and Ullu, 2010]. Defects in the retina and pronephros of zebrafish have been observed when a knockout of the $t t c$ 26 gene with a ciliary defect was performed [Zhang et al., 2012]. In mammalian cells, green fluorescent proteinfused TTC26 moves along the length of the cilia similar to many IFT proteins. GFP-fused TTC26 also plays a role in transporting specific ciliary proteins that are related to motility of the cilia or flagella [Ishikawa and Marshall, 2011]. Furthermore, knockout of ttc26/dyf13 in zebrafish embryos or mutations of TTC26/DYF13 in Chlamydomonas reinhardtii resulted in abnormal motility and short cilia [Ishikawa and Marshall, 2011].

In the present study, we report a Saudi proband who deceased at 15 months of age after severe clinical presentation of cholestasis, cystic dilatation of intrahepatic biliary ducts, diabetes insipidus, dysmorphic features, optic atrophy, pituitary hypoplasia, hydrocephalus, aqueduct stenosis, hyperextensible knee joints, and bilateral knee dislocation with polydactyly and syndactyly. Whole-genome sequencing (WGS) revealed a previously reported homozygous splice acceptor site variant of the TTC26 gene located in chromosome $7 \mathrm{q} 34$, providing evidence for the involvement of TTC26 in severe ciliopathy syndrome in humans.

\section{Materials and Methods}

Blood Mononuclear Cell Isolation and Study Approval

Whole blood samples were collected from the proband (II-2) and her unaffected parents (Fig. 1a), and peripheral blood mononuclear cells (PBMCs) were separated by density gradient centrifugation using Ficoll-prefilled Leucosep ${ }^{\circledR}$ tubes according to the manufacturer's instructions. PBMC percent viability was assessed using the trypan blue exclusion method (85-90\%).

\section{Genomic DNA Extraction}

Genomic DNA was extracted from fresh blood according to standard procedures using the QIAampDNA Micro kit, and quantification of the genomic DNA was performed according to standard methods using the NanoDrop ${ }^{\mathrm{TM}}$ spectrophotometer.

\section{Whole-Genome Sequencing}

WGS was conducted in the affected individual (II-2) as previously described [Alhamoudi et al., 2020]. Genomic DNA was fragmented using a sonicator. Illumina adapters were ligated with DNA fragments and subsequently sequenced on the HiSeqX platform (Illumina, San Diego, CA, USA) with an average coverage depth of approximately $30 \times$. Base calling was performed using an in-house designed pipeline, and primary filtering was performed to remove low-quality reads and artifacts. All disease-causing variants reported in $\mathrm{HGMD}^{\circledR}$, ClinVar, PubMed, and CentoMD ${ }^{\circledR}$ and variants with minor allele frequency of less than $1 \%$ in the ExAC/ gnom $A D$ database were given preference. Variant filtration steps also focused on coding exons and flanking $+/-20$ intronic bases [Alhamoudi et al., 2020].

Filtration of variants was performed by considering all patterns of inheritance. However, based on the autosomal recessive pattern of pedigree, homozygous and compound heterozygous variants were preferred. We focused on disease-causing nonsynonymous variants such as nonsense, missense, splice site variants, and frameshift coding insertions or deletions. In addition, clinical details of the patient were used to perform genotype-phenotype correlation.

\section{In silicoPrediction}

In silico prediction tools, such as Varsome, and MutationTaster, FATHMM-MKL, EIGEN, DANN, BayesDel addAF, EXSKIP, BDGP, NetGene 2 and GERP, were used to predict pathogenicity and its effect on splice site (Table 1).

\section{Sanger Sequencing}

The identified biallelic variant was Sanger sequenced bi-directionally in all available members of the family. Sanger sequencing was performed according to previously described methods [Umair et al., 2016]. Primer sequences were designed using Primer 3 online software (http://frodo.wi.mit.edu/primer3/).

\section{RNA Extraction}

Briefly, $1 \mathrm{~mL}$ of TRIzol ${ }^{\circledR}$ reagent was added to $\left(1 \times 10^{8}\right)$ PBMCs, and total RNA extraction was performed using standard methods. Then, $200 \mu \mathrm{L}$ of chloroform was added for the separation of the organic and aqueous phases. After centrifugation at $4^{\circ} \mathrm{C}$ for 15 min, RNA in the aqueous phase was transferred into an RNase-free tube. Washing was performed using isopropanol, and this was followed by precipitation with $75 \%$ ethanol and absolute ethanol. Total RNA was extracted from cultured fibroblasts $\left(10^{6}\right.$ cells) using the RNeasy Plus Mini Kit (Qiagen Inc., Valencia, CA, USA). Extracted RNA was analyzed for purity and quantified using standard methods.

\section{Quantitative Real-Time PCR}

Total RNA was extracted to quantitatively monitor TTC26 (NM_024926.3) mRNA expression relative to the internal control "house-keeping" gene GAPDH (DQ403057). cDNA was synthesized using a high capacity cDNA reverse transcription kit (Ap- 


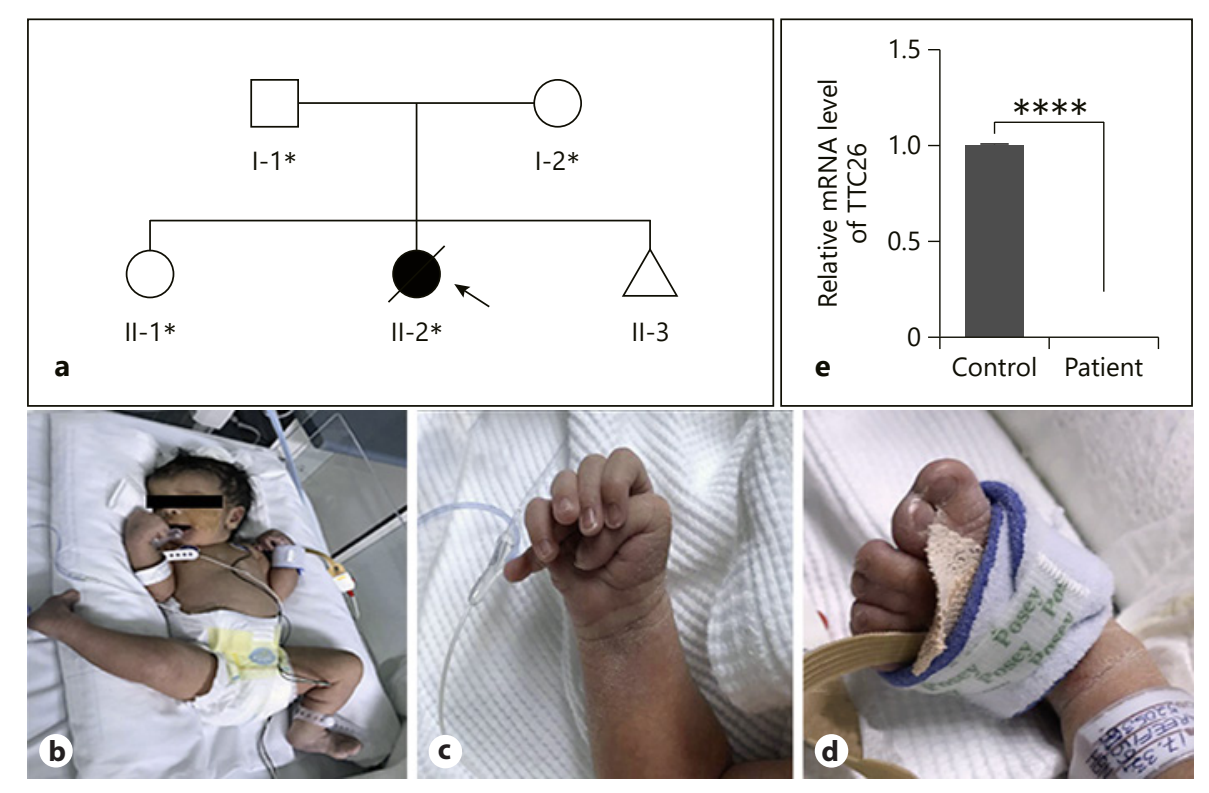

Fig. 1. a Pedigree of the family showing nonconsanguineous union and recessive inheritance pattern. White squares/circles represent unaffected individuals; the black circle represents the affected individual. Diagonal lines indicate deceased individual. Individuals labeled with asterisks were available for the present study. The triangle represents spontaneous abortion (pregnancy not carried to term). b Affected individual (II-2) presenting dysmorphic features in the form of deep-set eyes, low-set ears, frontal bossing, and hy- permobile and extensible joints. c Polydactyly in the upper limbs. d Complex syndactyly in the lower limbs. e The proband (II-2) revealed a significant decrease in human TTC26 gene expression as compared to the normal control. The bar graph shows the relative expression of TTC26 mRNA, which was determined using qRT-PCR. The results are presented as mean \pm standard deviation $(n=2)$, where $\left(^{*}\right)$ signifies a statistically significant difference $(p<$ $0.05)$ as compared to the control. plied Biosystems, Waltham, MA, USA). TTC26 gene amplification was performed by qPCR using primers TTC26-F1: $5^{\prime}$-GCTGTAGGCAGAGGCGTAC-3' and TTC26-R1: 5'-CACCCAGGTGAAAGGCAC- $3^{\prime}$. The primers were designed using the Primerbank database (https://pga.mgh.harvard.edu/primerbank/index. html). The qPCR reaction was performed using a PCR SYBR Green Master Mix (Thermo Fisher, Waltham, MA, USA) on a 7500 real-time PCR system (Thermo Fisher). All reactions were performed in triplicate and repeated independently. ExpressionSuite software version 1.1 (Applied Biosystems) was used for analysis [Asiri et al., 2020; Umair et al., 2020].

The qPCR results are expressed as mean \pm standard deviation. A minimum of 3 independent experiments were performed and data were collected. To compare the 2 groups, an unpaired Student's $t$-test was performed and a value of $p<0.05$ was considered to be significant.

\section{Results}

\section{Clinical Description}

A Saudi female infant was born to nonconsanguineous healthy parents at full term by cesarean section due to fetal distress. The birth weight was $2.7 \mathrm{~kg}$ (5th percentile),
Table 1. Tools used to validate and predict splice site variant

\begin{tabular}{lll}
\hline S.No & Tool used & Prediction \\
\hline 1 & MutationTaster & Disease causing \\
2 & Varsome & Pathogenic \\
3 & EIGEN & Pathogenic \\
4 & FATHMM-MKL & Damaging \\
5 & BayesDel addAF & Damaging \\
6 & EX-SKIP & Exon skipping (pathogenic) \\
7 & BDGP & Splice site changed (pathogenic) \\
8 & NetGene2 & Splice site changed (pathogenic) \\
9 & GERP & Damaging \\
\hline
\end{tabular}

birth length was $47 \mathrm{~cm}$ (5th percentile), and birth head circumference was $35 \mathrm{~cm}$ (50th percentile). Antenatally, she was found to have polyhydramnios and atrioventricular septal defect. She was born non-vigorous with Apgar scores of 5, 7, and 7 at 1, 5, and 10 min, respectively, and she had a weak cry and hypotonia. Therefore, the patient was resuscitated and shifted to the neonatal intensive care unit. During her stay in the care unit, she was provided 


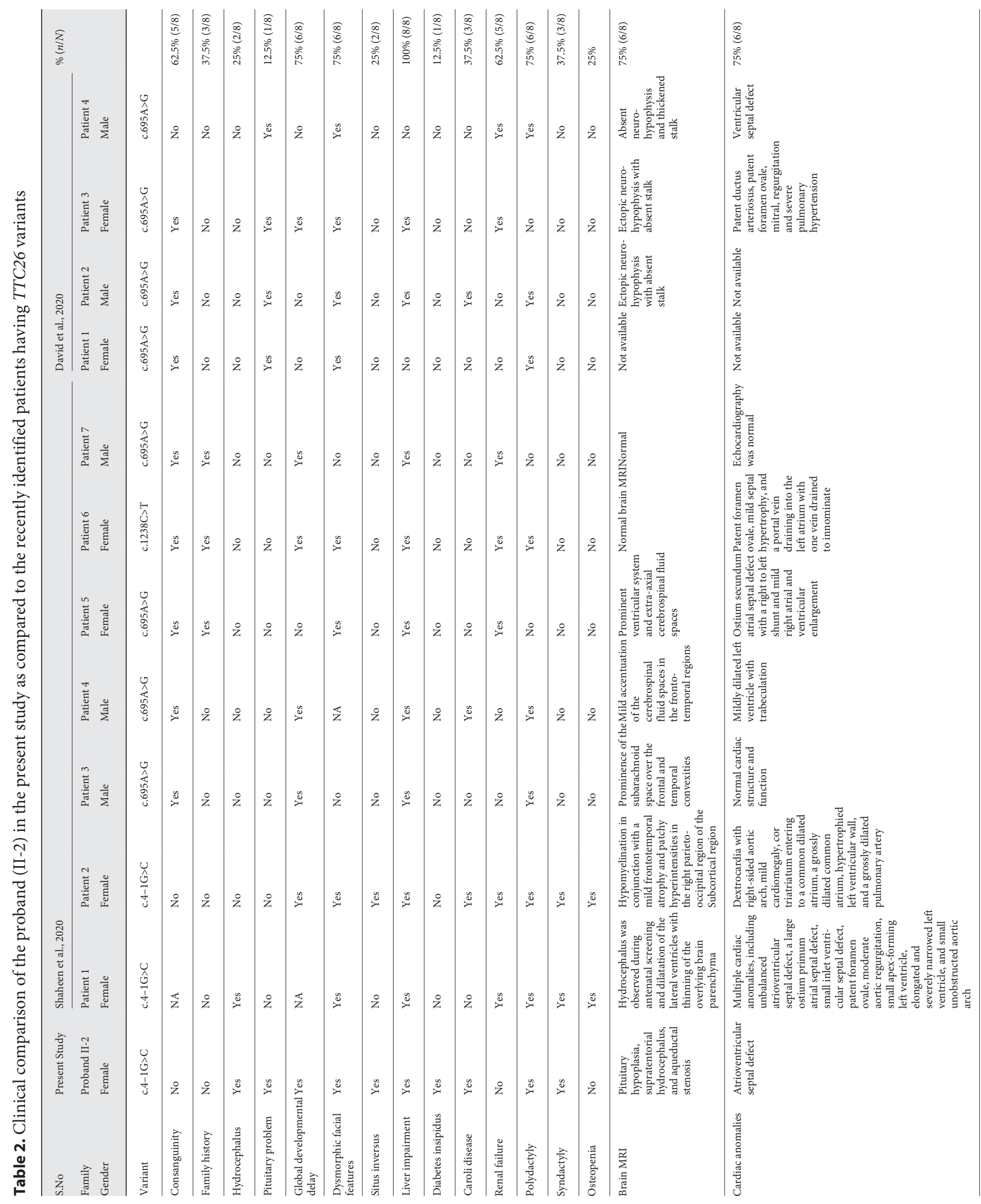




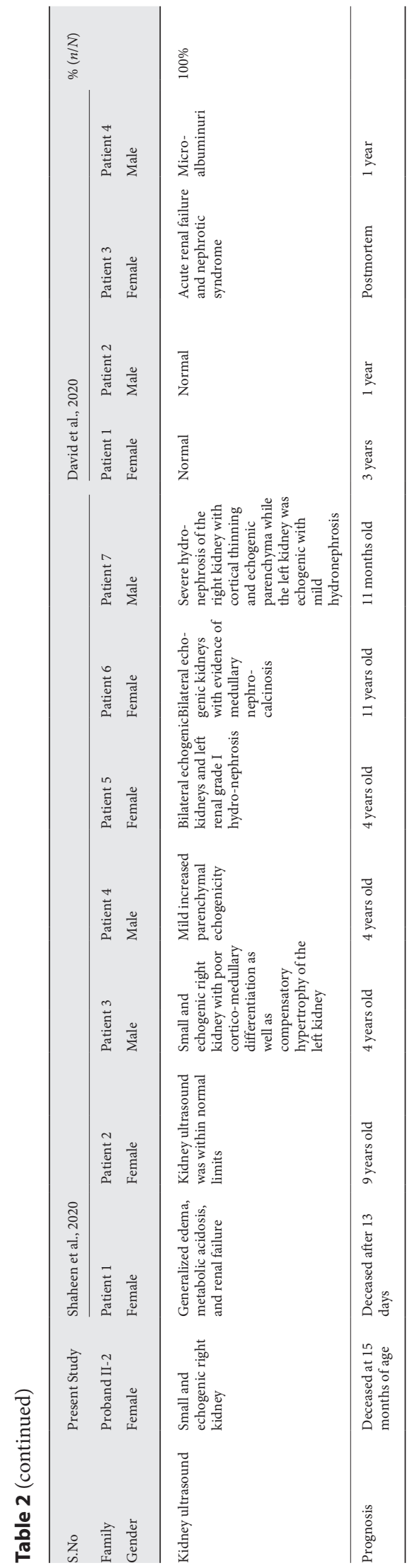

Mutated TTC26 Causes BRENS Syndrome respiratory support with nasal continuous positive airway pressure, and was found to have dysmorphic features in the form of deep-set eyes, low-set ears, frontal bossing, hypermobile and extensible joints, polydactyly in the upper limbs, and syndactyly in the lower limbs (Fig. 1b-d). Echocardiography was performed immediately, and it showed a large primum atrial septal defect with a left-toright shunt and patent ductus arteriosus that closed spontaneously later on. Additionally, she suffered from cholestatic jaundice; ultrasound of the abdomen showed persistent cystic dilatation of the intrahepatic biliary ducts, which was suspected to be Caroli disease. Liver biopsy demonstrated zone 3 cholestasis with ductopenia and mild ductular reaction. The patient was administered ursodiol $28 \mathrm{mg}$ b.i.d.; however, no improvement was observed. From an endocrine point of view, she had diabetes insipidus, which was treated with desmopressin. Brain MRI showed pituitary hypoplasia.

Additionally, supratentorial hydrocephalus and aqueductal stenosis were observed, and ventriculoperitoneal shunt insertion was performed to manage them. Neurologically, she had global developmental delay and axial hypotonia. From a musculoskeletal point of view, she had hyperextensible joints, specifically congenital bilateral knee dislocation (more toward right than left), which improved after serial knee casting. Moreover, her right kidney was small and echogenic with normal function. She was discharged and subsequently admitted multiple times with recurrent respiratory infections, aspiration pneumonia, and severe gastroesophageal reflux. Gastrostomy insertion with fundoplication was performed to manage the gastroesophageal reflux. Failure to thrive was observed with severe global developmental delay at 3 months of age and axial hypotonia. Eye examination showed right congenital third nerve palsy with widening of the optic cup. No edema or coloboma was observed, and the hearing test was normal. She continued to have persistent central fever, reached a vegetative state, and deceased at 15 months of age with cardiorespiratory arrest. We did not test the aborted fetus (II-3), thus it might be possible that the ciliopathy syndrome seen in proband (II-2) caused the embryo lethality in the aborted fetus (II-3).

Detailed clinical descriptions of the proband reported in this study are presented in Table 2.

\section{WGS and Sanger Sequencing}

WGS identified a homozygous splice acceptor site variant in the TTC26 gene (NM_024926.3; g.138819400G $>$ C \{GRCh38/hg38\}; c.4-1G $>$ C) located in chromosome $7 \mathrm{q} 34$ that cosegregated with the disease 
phenotype and was verified using Sanger sequencing. The splice site variant (c.4-1G>C) has been reported to be pathogenic, and it is predicted to affect all 6 protein-coding transcripts of TTC26. The identified variant was not observed in the homozygous state in the in-house (1410) Saudi exome, Saudi genome database (https://sso.saudigenomeproject.com/accounts/login/?next =/), ExAC, or gnom $A D$ database. The pathogenicity index of the identified variant was calculated using different available online tools, and the identified splice acceptor site variant was considered pathogenic. The identified variant is classified as class $2 \mathrm{P}$ (likely affecting protein function) according to the American College of Medical Genetics and Genomics guidelines.

\section{Quantitative Real-Time PCR}

Using qPCR, the TTC26 gene mRNA expression was investigated in the proband and a normal control individual. The proband (II-2) revealed a significant decrease in the relative gene expression of human TTC26, as compared to normal control (Fig. 1e).

\section{Discussion}

Shaheen et al. [2020] and David et al. [2020] described individuals with severe ciliopathy phenotypes, including neonatal cholestasis, in different Saudi and Israeli families. In the present study, we investigated a Saudi proband with severe clinical presentations such as optic atrophy, cholestasis, knee hyperextensibility, hydrocephalus, atrioventricular septal defect, pituitary hypoplasia, and developmental delay. Features such as developmental delay, dysmorphic facial features, liver abnormalities, and cardiovascular disorders were similar to those reported previously [Shaheen et al., 2020]. More recently, pituitary stalk interruption syndrome has been reported in patients [David et al., 2020]. Pituitary hypoplasia was also reported in our patient; however, diabetes insipidus was not reported previously, and hydrocephalus has been reported in 1 of the 7 ciliopathies cases so far.

Sequence variations in several ciliopathy genes have been associated with hydrocephalus. Biallelic variations in different ciliopathy genes, such as CC2D2A (OMIM 612013), TMEM67 (OMIM 609884), and MKS1 (OMIM 609883), have been associated with clinical presentations, such as hydrocephalus. Motile cilia on ependymal cells of the brain exhibit motion and help in fluid movement. The proper movement of cilia helps in the flow of cerebrospinal fluid, and the disruption of proper movement (beat- ing) by irregular planar cell polarity causes the accumulation of cerebrospinal fluid resulting in hydrocephalus [Faubel et al., 2016]. Similarly, variations in the WDR11 gene have been associated with ciliopathy-related disorders and Kallmann syndrome. Associated features include pituitary dysgenesis and obesity [Kim et al., 2018].

Using WES, Shaheen et al. [2020] identified 3 homozygous variants (c.695A $>\mathrm{G} ; \mathrm{c} .4-1 \mathrm{G}>\mathrm{C} ; \mathrm{c} .1238 \mathrm{C}>\mathrm{T}$ ) in the TTC26 gene of 7 different families in Saudi. Recently, David et al. [2020] identified a homozygous missense variant, c.695A>G (p.Asn232Ser), in 2 Israeli families.

In this study, we identified a previously reported homozygous splice site variant (c.4-1G>C) in the TTC26 gene located in chromosome $7 \mathrm{q} 34$. The variant is pathogenic in nature because $\mathrm{cDNA}$ sequencing previously revealed skipping of exon 2 on TTC26, which resulted in the deletion of 45 amino acids downstream (p.Met2_Glu$47 \mathrm{del}$ ) [Shaheen et al., 2020]. The skipping of exon 2 decreases the size of the final protein, which might be a result of the canonical splicing of exon 1 by knocking out its natural $3^{\prime}$ acceptor splice site and eliciting the use of a cryptic splice site, which led to the loss of 45 amino acids.

Using RNA samples from the patient, qRT-PCR revealed a substantial decrease in the expression of TTC26 in the proband as compared to the normal control (Fig. 1e).

Almost 187 genes have been reported to cause 35 different ciliopathies, and more than 241 genes have been associated with ciliary structure and function in humans. Cilia assembly is a complex process that includes the attachment of the centriole to the plasma membrane and a diffusion barrier, which distinguishes the cilia from rest of the cell [Sung and Leroux, 2013]. Later, the growth of the axoneme, which helps in the transportation of the building blocks from the protein synthesis site (cytoplasm) to the tip of the axoneme, involves an active process called IFT [Kozminski t al., 1993]. Motor kinesin II facilitates the movement of IFT proteins from the base to the tip of the axoneme, where they are remodeled. The exact composition of IFT is not known; however, it is composed of IFT$A$ and IFT-B complexes [Piperno and Mead, 1997]. The IFT-B mutant complex gives rise to no cilia or very short cilia [Jonassen et al., 2008; Tsao and Gorovsky, 2008], whereas the mutant IFT-A results in swollen cilia with IFT-B particles [Qin et al., 2011; Liem et al., 2012]. It has been observed that IFT-A arbitrates retrograde transport, while IFT-B mediates anterograde transport.

Until now, only a few IFT proteins have been functionally characterized, such as IFT25, which is involved in $\mathrm{SHH}$, although it is not necessary for cilia assembly [Ke- 
ady et al., 2012] and IFT46, which plays a role in the transportation of the outer dynein arms into the flagella [Ahmed et al., 2008]. Polyglutamylation of axonemal tubulin is performed by specific IFT70 proteins, and at the tip of the flagella, IFT172 functions by interchanging between retrograde and anterograde transport [Pedersen et al., 2005]. Furthermore, the N-terminals of IFT81 and IFT74 form a tubulin-binding module and IFT56 (IFT-B subunit) functions in the transportation of the inner dynein arm subunits [Bhogaraju et al., 2013; Ishikawa et al., 2014].

TTC26, also known as IFT56, is a component of the IFTB complex, and its deficiency in several model organisms has regularly shown defective ciliary function. In TTC26 mutants, cilia display impaired function and variable length, suggesting dysregulation of SHH and IFT-B component abnormalities [Shaheen et al., 2020]. In an embryonic mouse, high expression of Ttc26has been observed in the liver, suggesting its key role in the normal development of the intrahepatic biliary system [Shaheen et al., 2020].

In conclusion, we reported a Saudi family with a complex ciliopathy disorder due to an already reported pathogenic variant in the TTC26 gene. Our study provides additional evidence that mutated/missing TTC26 leads to abnormal ciliary development in humans. We named this newly characterized syndrome as BRENS syndrome, which stands for biliary, renal, neurological, and skeletal features.

\section{Acknowledgments}

We are grateful to the patient's family for their compliance and support. Permission was obtained from the patient's parents for presentation of images and publication of this report.

\section{Statement of Ethics}

All procedures were approved by the research committee of King Abdullah International Medical Research Center (KAIMRC), Riyadh, Saudi Arabia and were performed in accordance with the ethical standards. Written informed consent was obtained from the patient's parents for publication of this case report and any accompanying images.

\section{Conflict of Interest Statement}

The authors have no conflicts of interst to declare.

\section{Funding Sources}

This work was funded by the King Abdullah International Medical Research Center, project number: RC18/017/R.

\section{Author Contributions}

M.U. and B.A. drafted the manuscript. A.A., B.A., K.A., and M.O. collected samples and clinical data, analyzed the data, and performed experiments. A..T, Y.A. analyzed the data. M.A. was responsible for conception, study design and editing the manuscript. All the authors read and approved the final manuscript.

\section{Web Sources}

NetGene2: http://www.cbs.dtu.dk/services/NetGene2/ BDGP: Berkeley Drosophila Genome Project, https://www. fruitfly.org/about/index.html

EX-SKIP: https://ex-skip.img.cas.cz/

MutationTaster: http://www.mutationtaster.org/

FATHMM-MKL: http://fathmm.biocompute.org.uk/ fathmmMKL.htm.

DANN: https://cbcl.ics.uci.edu/public_data/DANN/

BayesDel addAF: http://fengbj-laboratory.org/BayesDel/ BayesDel.html.

Varsome: https://varsome.com/

EIGEN: http://www.columbia.edu/ ii2135/eigen.html.

OMIM: https://omim.org/

Ensembl: http://ensembl.org/index.html.

UCSC Genome Browser Home: https://genome.ucsc.edu/

GERP: http://mendel.stanford.edu/SidowLab/downloads/gerp/ index.html.

\section{References}

Ahmed NT, Gao C, Lucker BF, Cole DG, Mitchell DR. ODA16 aids axonemal outer row dynein assembly through an interaction with the intraflagellar transport machinery. J Cell Biol. 2008;183(2):313-22.

Alhamoudi KM, Bhat J, Nashabat M, Alharbi M, Alyafee Y, Asiri A, et al. A Missense Mutation in the UGDH Gene Is Associated With Developmental Delay and Axial Hypotonia. Front Pediatr. 2020;8:71.
Asiri A, Aloyouni E, Umair M, Alyafee Y, Al Tuwaijri A, Alhamoudi KM, et al. Mutated RAP1GDS1 causes a new syndrome of dysmorphic feature, intellectual disability \& speech delay. Ann Clin Transl Neurol. 2020;7(6): 956-64.

Bhogaraju S, Cajanek L, Fort C, Blisnick T, Weber $\mathrm{K}$, Taschner M, et al. Molecular basis of tubulin transport within the cilium by IFT74 and IFT81. Science. 2013;341(6149):1009-12.
David O, Eskin-Schwartz M, Ling G, Dolgin V, Kristal E, Benkowitz E, et al. Pituitary stalk interruption syndrome broadens the clinical spectrum of the TTC26 ciliopathy. Clin Genet. 2020;98(3):303-7.

Faubel R, Westendorf C, Bodenschatz E, Eichele G. Cilia-based flow network in the brain ventricles. Science. 2016;353(6295):176-8.

Follit JA, Xu F, Keady BT, Pazour GJ. Characterization of mouse IFT complex B. Cell Motil Cytoskeleton. 2009;66(8):457-68. 
Franklin JB, Ullu E. Biochemical analysis of PIFTC3, the Trypanosoma brucei orthologue of nematode DYF-13, reveals interactions with established and putative intraflagellar transport components. Mol Microbiol. 2010; 78(1):173-86.

Hildebrandt F, Benzing T, Katsanis N. Ciliopathies. N Engl J Med. 2011;364(16):1533-43.

Ishikawa $\mathrm{H}$, Marshall WF. Ciliogenesis: building the cell's antenna. Nat Rev Mol Cell Biol. 2011;12(4):222-34.

Ishikawa $\mathrm{H}$, Ide T, Yagi T, Jiang X, Hirono M, Sasaki H, et al. TTC26/DYF13 is an intraflagellar transport protein required for transport of motility-related proteins into flagella. ELife. 2014;3:e01566.

Jonassen JA, San Agustin J, Follit JA, Pazour GJ. Deletion of IFT20 in the mouse kidney causes misorientation of the mitotic spindle and cystic kidney disease. J Cell Biol. 2008;183(3): 377-84.

Keady BT, Samtani R, Tobita K, Tsuchya M, San Agustin JT, Follit JA, et al. IFT25 links the signal-dependent movement of Hedgehog components to intraflagellar transport. Dev Cell. 2012;22(5):940-51.

Kim YJ, Osborn DP, Lee JY, Araki M, Araki K, Mohun T, et al. WDR11囚mediated Hedgehog signalling defects underlie a new ciliopathy related to Kallmann syndrome. EMBO Rep. 2018;19(2):269-89.
Kozminski KG, Johnson KA, Forscher P, Rosenbaum JL. A motility in the eukaryotic flagellum unrelated to flagellar beating. Proc Natl Acad Sci USA. 1993;90(12):5519-23.

Liem KF, Ashe A, He M, Satir P, Moran J, Beier $\mathrm{D}$, et al. The IFT-A complex regulates Shh signaling through cilia structure and membrane protein trafficking. J Cell Biol. 2012;197(6): 789-800.

Pedersen LB, Miller MS, Geimer S, Leitch JM, Rosenbaum JL, Cole DG. Chlamydomonas IFT172 is encoded by FLA11, interacts with CrEB1, and regulates IFT at the flagellar tip. Curr Biol. 2005;15(3):262-6.

Piperno G, Mead K. Transport of a novel complex in the cytoplasmic matrix of Chlamydomonas flagella. Proc Natl Acad Sci USA. 1997;94(9): 4457-62.

Qin J, Lin Y, Norman RX, Ko HW, Eggenschwiler JT. Intraflagellar transport protein 122 antagonizes Sonic Hedgehog signaling and controls ciliary localization of pathway components. Proc Natl Acad Sci USA. 2011;108(4): 1456-61.

Reiter JF, Leroux MR. Genes and molecular pathways underpinning ciliopathies. Nat Rev Mol Cell Biol. 2017;18(9):533-47.

Scholey JM. Intraflagellar transport motors in cilia: moving along the cell's antenna. J Cell Biol. 2008;180(1):23-9.

Shaheen R, Szymanska K, Basu B, Patel N, Ewida $\mathrm{N}$, Faqeih E, et al. Characterizing the morbid genome of ciliopathies. Genome Biol. 2016; 17(1):242
Shaheen R, Alsahli S, Ewida N, Alzahrani F, Shamseldin HE, Patel N, et al. Biallelic Mutations in Tetratricopeptide Repeat Domain 26 (Intraflagellar Transport 56) Cause Severe Biliary Ciliopathy in Humans. Hepatology. 2020;71(6):2067-79.

Sung CH, Leroux MR. The roles of evolutionarily conserved functional modules in cilia-related trafficking. Nat Cell Biol. 2013;15(12):138797.

Tsao CC, Gorovsky MA. Tetrahymena IFT122A is not essential for cilia assembly but plays a role in returning IFT proteins from the ciliary tip to the cell body. J Cell Sci. $2008 ; 121(\mathrm{Pt} 4)$ : $428-36$.

Umair M, Hassan A, Jan A, Ahmad F, Imran M, Samman MI, et al. Homozygous sequence variants in the FKBP10 gene underlie osteogenesis imperfecta in consanguineous families. J Hum Genet. 2016;61(3):207-13.

Umair M, Ballow M, Asiri A, Alyafee Y, Tuwaijri AA, Alhamoudi KM, et al. EMC10 Homozygous Variant Identified in a Family with Global Developmental Delay, Mild Intellectual Disability, and Speech Delay. Clin Genet. 2020;98(6):555-61.

Zhang Q, Liu Q, Austin C, Drummond I, Pierce EA. Knockdown of ttc26 disrupts ciliogenesis of the photoreceptor cells and the pronephros in zebrafish. Mol Biol Cell. 2012;23(16):306978. 